\title{
Simulation and analysis of microwave heating while joining bulk copper
}

\author{
M. S. Srinath ${ }^{1 *}$, P. Suryanarayana Murthy², Apurbba Kumar Sharma ${ }^{3}$, \\ Pradeep Kumar ${ }^{4}$, M. V. Kartikeyan ${ }^{5}$ \\ ${ }^{\prime *}$ Department of Mechanical Engineering, Malnad College of Engineering, Hassan-573201, INDIA \\ ${ }^{2,3,4}$ Department of Mechanical and Industrial Engineering, Indian Institute of Technology Roorkee, Roorkee - 247 667, INDIA. \\ ${ }^{5}$ Department of Electronics and Computer Science Engineering, Indian Institute of Technology Roorkee, Roorkee - 247 667, INDIA. \\ ${ }^{*}$ Corresponding Author: e-mail: ${ }^{1 *}$ Srinadme@gmail.com Tel+19-8277421917
}

\begin{abstract}
Processing of bulk metallic materials using microwave energy is challenging. However, in this work microwave heating has been successfully employed for joining of bulk copper. The present work illustrates the joining process using COMSOL simulation tool. The work complements the experimental results of the microwave heating of bulk copper in a multimode microwave applicator. In electromagnetic processing, thermal dissipation in the bulk metal may be attributed to resistive heating, dielectric and magnetic losses. This dissipative mechanism is coupled to the fields by the conductivity, permittivity and permeability of the metal. The model has been created for heating of the bulk metal in a microwave oven by considering the radio frequency of the microwaves. The distributed power and heat source were computed in a stationary, frequency domain electromagnetic analysis and coupled to a transient heat transfer simulation showing the heat distribution in the bulk copper joint. The maximum power observed at the joint interface is $6.0618 \times 10^{8} \mathrm{~W} / \mathrm{m}^{3}$ with an average power is $3 \times 10^{8} \mathrm{~W} / \mathrm{m}^{3}$. However, the temperature will be around $1144^{\circ} \mathrm{C}$ was attained in $300 \mathrm{~s}$ at the joint interface while the parent material is about $940{ }^{\circ} \mathrm{C}$. The simulation results are in good agreement with the experimental one.
\end{abstract}

Keywords: Microwave heating, FEM Model, Simulation, Copper Joint, Resistive Heating.

DOI: http://dx.doi.org/10.4314/ijest.v4i2.11

\section{Introduction}

Heating of a material using electromagnetic energy is based on a material's capacity to efficiently absorb set energy. It is quite possible to heat a range of materials rapidly through microwave heating methods. In comparison to conventional heating methods, microwave processing has the possibility of increased energy efficiency. By transferring energy through microwaves, heat transfer is not only limited on the surface of a material, but is transferable to entire volume and allowing for increased rates of heat transfer. As energy is transferred using electromagnetic waves, a new temperature profile exists for microwave processing due to the ability to penetrate inner surface layers. The heating is not dependent upon surface area, but dependent upon volume; hence an inverse heating profile is present (Sutton, 1989, Clark et al., 1996). The use of microwaves to process engineering materials has been started in 1970s and applied to a wide variety of materials including wood, foodstuffs, rubber, ceramics, semiconductors and metals subsequently. Microwave processing of materials can result in materials with different microstructures and enhanced properties compared with conventional sintering (Sharma et al., 2001). Moreover, microwave processing requires much less energy than conventional methods. Although microwave technology has been used in processing of materials for many years, its mechanism is still not well understood, especially regarding the effects of the magnetic field of the electromagnetic wave.

Literature indicates that, the dielectric properties are considered as main heating source during microwave processing of materials and the contribution from magnetic energy losses are generally ignored. However, recent experimental results showed that the magnetic field also plays an important role during microwave processing, especially for sintering of ferromagnetic/magnetic materials and semimetal/ metal composite materials (Agarwal et al., 1999; Ayappa et al., 1991). For 
sintering these materials, by separating the electric field $(\mathbf{E})$ and magnetic field $(\mathbf{H})$, the experiments revealed that the magnetic field $\mathbf{H}$ can also play an important role in the heating process (Chatterjee et al., 1998). Unfortunately, there are no theoretical studies or simulations that consider both contributions from $\mathbf{E}$ and $\mathbf{H}$ fields in microwave heating. Instead, most of the previous theoretical work just treated sintering by considering only the dielectric contributions and assumed that the contributions from magnetic energy losses are very small and consequently can be ignored (Olevsky, 1998; Yuhua et al., 2006; Ma et al., 2006). Available literature shows that considerable work has been carried out in the area of simulation of sintering of metallic materials. However, no reports are available on theoretical studies on brazing/joining of metallic materials. Further, selective heating is of significance in microwave processing of materials. During experimentation the selective heating characteristics of microwave processing cannot be visualized, thus, theoretical studies or simulation of the process is required. The present article illustrates a model for the joining of bulk copper using interface powder layer through microwave energy and a finite element simulation with the COMSOL Multiphysics. The distributed heat source is computed in a stationary, frequency domain electromagnetic analysis followed by a transient heat transfer simulation showing the temperature redistribution in the joint interface.

\section{Modeling and formulation}

In the present work three dimensional (3D) microwave heating geometries were created in the similar manner which has been used during experimentation. The microwave chamber is a metallic box with a $1 \mathrm{~kW}, 2.45 \mathrm{GHz}$ microwave source via a rectangular waveguide operating in the $\mathrm{TE}_{10}$ mode, which is ideal in the present work. Cylindrical glass plate is placed at the bottom of the oven, on top of it copper plates along with interface material is placed, which is similar to experimental process. The symmetry cut is applied vertically through oven, waveguide, copper plates and the cylindrical plate for simulating only half of the problem. Front view of the model and their dimensions are shown in Fig 1.

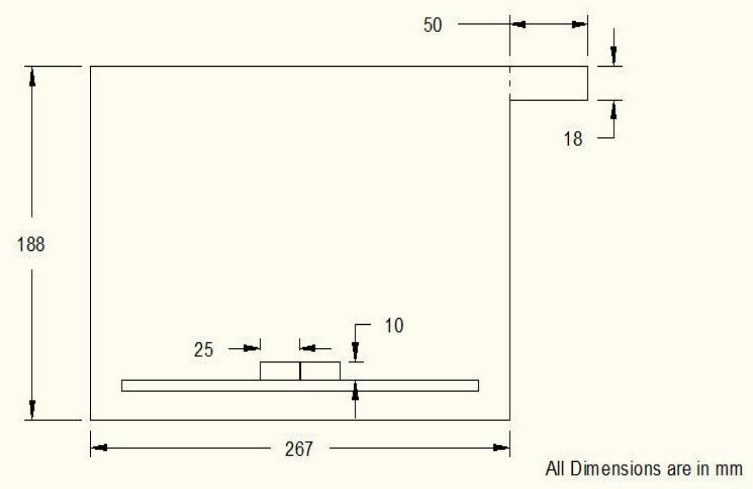

Fig 1. Typical Front view of the simulated model

Figure 2(a) illustrates simulation model, showing a simplified version of the experimental setup used during experiments. The copper specimens to be joined are $25 \mathrm{~mm} \times 10 \mathrm{~mm} \times 10 \mathrm{~mm}$ and the interface layer have the thickness of $1 \mathrm{~mm}$.The reduced geometry along with fine and coarse meshing is shown in Fig. 2(b). It is observed from Fig. 2(b) that fine meshing has been created in the area where electromagnetic interaction is required and remaining areas are meshed with coarser one.
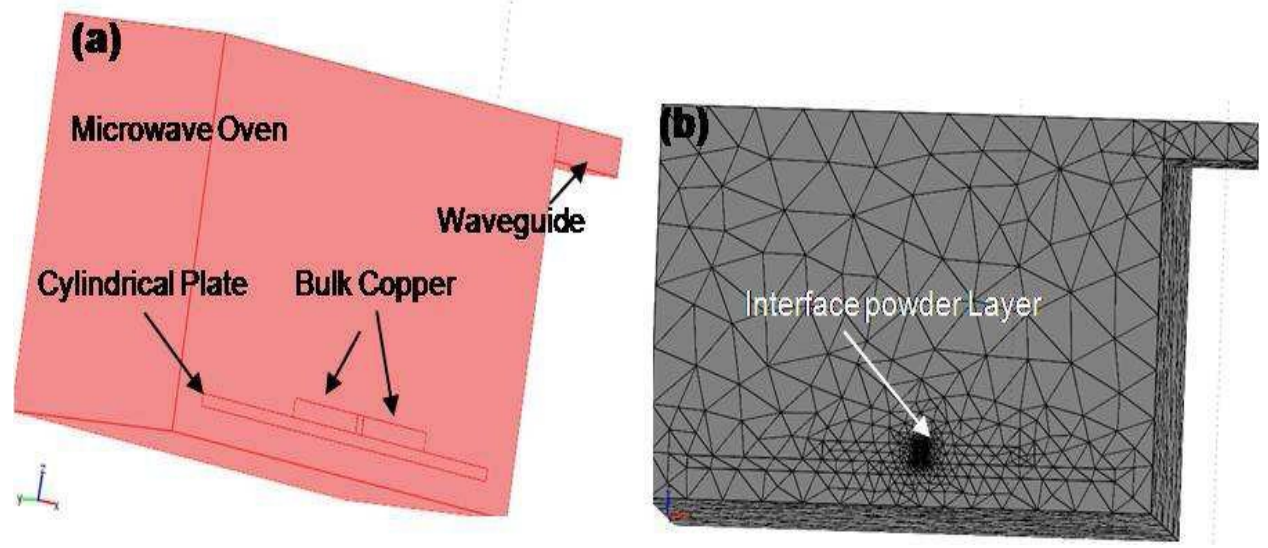

Fig. 2 (a) FEM Model of the bulk Copper joint; (b) Meshing of the copper joint for microwave heating. 
The walls of the oven and the waveguide are treated as good conductors. The model approximates these walls as perfect conductors, represented by the boundary condition $\mathrm{n} \times \mathrm{E}=0$. The symmetry cut has mirror symmetry for the electric field and is represented by the boundary condition $\mathrm{n} \times \mathrm{H}=0$. For the electromagnetic propagation and heat transfer modules, the boundary conditions are different. In the heat transfer module, the heat is transferred from the interface layer to the bulk interfaces, and then to the air; therefore, the boundary condition between the interface layer and air was taken to be the heat flux. In the electromagnetic module the electromagnetic wave from waveguide in a 3D model while the boundary conditions were set to port options. The boundary between the bulk copper, interface layer and the air is taken as a perfect conductor as shown in Fig. 1. Since the enclosure atmosphere can influence the thermal and electrical properties of the interface layer, for simplicity, air atmosphere is considered in all simulations. Combination of the propagation equation of the electromagnetic waves $\left(\mathrm{TM}_{01}\right.$ or $\mathrm{TE}_{10}$ mode) with the heat transfer equation is used in order to get the temperature distributions and other system properties. The rectangular port is excited by a transverse electric (TE) wave, which has no electric field component in the direction of propagation. At an excitation frequency of $2.45 \mathrm{GHz}$, the $\mathrm{TE}_{10}$ mode is the only propagating mode through the rectangular waveguide. The cutoff frequencies for the different modes are given analytically by the relation (1),

$$
\left(v_{c}\right)_{\mathrm{mn}}=\frac{c}{2} \sqrt{\left(\left(\frac{m}{a}\right)^{2}-\left(\frac{n}{b}\right)^{2}\right)}
$$

where $\mathrm{m}$ and $\mathrm{n}$ are the mode numbers and $\mathrm{c}$ denotes the speed of light. For the $\mathrm{TE}_{10}$ mode, $\mathrm{m}=1$ and $\mathrm{n}=0$. With the dimensions of the rectangular cross section $(\mathrm{a}=7.8 \mathrm{~cm}$ and $\mathrm{b}=1.8 \mathrm{~cm})$, the $\mathrm{TE}_{10}$ mode is used as propagating mode for frequencies between 1.92 $\mathrm{GHz}$ and $3.84 \mathrm{GHz}$. With the stipulated excitation at the rectangular port, the following equation (2) is solved for the electric field vector $\mathrm{E}$ inside the waveguide and oven,

$$
\nabla \times\left\{\mu_{r}^{-1} \cdot \nabla \times E\right\}-\omega^{2}\left(\varepsilon_{r}-j\left(\frac{\sigma}{\omega \cdot \varepsilon_{o}}\right)\right) E=0
$$

where $\mu_{\mathrm{r}}$ denotes the relative permeability, $\mathrm{j}$ the imaginary unit, $\sigma$ the conductivity, $\omega$ the angular frequency, $\varepsilon_{\mathrm{r}}$ the relative permittivity, and $\varepsilon_{0}$ the permittivity of free space. The permittivity $\left(\varepsilon_{\mathrm{r}}\right)$ and permeability $\left(\mu_{\mathrm{r}}\right)$ are complex parameters and are temperature and frequency dependent. By applying standard boundary conditions and using finite element methods, one can solve equation (2) to obtain the distribution of the $\mathbf{E}$ and $\mathbf{H}$ fields. The power flux associated with propagating electromagnetic waves is represented by the Poynting vector $\mathbf{S}$ which is defined as (3),

$$
S=\frac{E \times H}{2}
$$

The Poynting theorem allows the evaluation of the electromagnetic power dissipated in the medium. This dissipated power is the total heating source in the heating process. The total heating source $(q)$ includes the joule resistive loss and the electromagnetic power dissipated per unit volume, as given by the following relation (4),

$$
q=\sigma E \cdot E^{*}-\operatorname{Re}(\nabla \cdot S)=\sigma E \cdot E^{*}+\frac{1}{2} \omega\left(\varepsilon_{r} E \cdot E^{*}+\mu_{r} H \cdot H^{*}\right)
$$

The port is a passage to allow the microwaves to pass through and enter into the applicator. In the present simulation, the port is assumed to be operating in transverse electric (TE10) mode only. In order to validate the simulation results, the input power used in the present study was maintained the same throughout as that of the power used during experimentation. The boundary conditions used for the present analyses are as shown in Table 1.

Table 1 Port boundary conditions used at the wave guides during microwave heating

\begin{tabular}{|l|l|}
\hline Port number & 1 \\
\hline $\mathrm{P}_{\text {in }}$ & $900 \mathrm{~W}$ \\
\hline Mode type & Transverse electric $\left(\mathrm{TE}_{10}\right)$ \\
\hline $\mathrm{E}_{0}$ & $0,0, \cos \left(\mathrm{pi}^{*}(\mathrm{y}) / 7.8[\mathrm{~cm}]\right)$ \\
\hline
\end{tabular}

The various material parameters used in the present model for transverse electric mode are as shown in Table 2. The interface powders has a mixture of both copper (core) and copper oxide (shell) and its relative permittivity is measured to be 10.2 [Chaterjee et al, 2005]. It was observed during experimental results that the joint will have mixture of both copper and copper oxide and one of the study confirmed that nearly $26 \%$ of copper oxide is present in the joint area [Srinath et al, 2011]. Accordingly, the value of permittivity has been considered in the present study. The permittivity of mixture is given by the relation (5), 
Table 2 Parametric inputs for transverse electric mode

\begin{tabular}{|c|c|c|c|c|}
\hline Properties & Air & Glass plate & $\begin{array}{c}\text { Mixture of } \\
\text { copper and } \\
\text { copper oxide [10] }\end{array}$ & Bulk copper \\
\hline Conductivity $(\sigma)$ & 0 & $1 \mathrm{e}-14$ & $1.5 \mathrm{e}-3$ & $5.99 \mathrm{e} 7$ \\
\hline Permittivity $\left(\varepsilon_{\mathrm{r}}\right)$ & 1 & 4.2 & $3.392-\mathrm{i}^{*} 0.055$ & 1 \\
\hline Permeability $\left(\mu_{\mathrm{r}}\right)$ & 1 & 1 & 1 & 1 \\
\hline \multicolumn{5}{c}{$\varepsilon_{\text {reffective }}=1+\frac{\left(\varepsilon_{r}-1\right) N A_{s}}{A}$} \\
$\varepsilon_{\text {reffective }}=3.392$
\end{tabular}

Where, $\left(\mathrm{NA}_{\mathrm{s}} / \mathrm{A}\right)$ is percentage of $\mathrm{CuO}$ in the mixture

The heat transfer can be described by following equation (6):

$$
\sigma C_{p}\left(\frac{d T}{d t}\right)-\nabla \cdot k \nabla T=q
$$

where $\rho$ is the density $\mathrm{Cp}$ is the heat capacity, $\mathrm{k}$ is the heat transfer coefficient, $\mathrm{T}$ is temperature and $\mathrm{t}$ is the time. By solving equation (6) with certain boundary conditions the heating temperature (T) distributions in the sample as function of the simulation time $t$ can be obtained. The various properties of copper used in the present study are as shown in Table 3 .

Table 3 Properties of copper for thermal analysis

\begin{tabular}{|c|c|c|}
\hline $\begin{array}{l}\text { Conductivity } \\
(\mathrm{W} /(\mathrm{m} \cdot \mathrm{k})\end{array}$ & $\begin{array}{l}\text { Heat capacity } \\
\left(\mathrm{c}_{\mathrm{p}}\right) \mathrm{J} /(\mathrm{kg} \cdot \mathrm{k})\end{array}$ & $\begin{array}{l}\text { Density } \\
(\rho) \mathrm{kg} / \mathrm{m}^{3}\end{array}$ \\
\hline 400 & 385 & 8940 \\
\hline
\end{tabular}

\section{Results and discussion}

Figure 3 shows the distributed microwave power source as a slice plot through the center of the interface powder layer. It is observed from the figure that maximum power of $6.069 \times 108 \mathrm{~W} / \mathrm{m} 3$ was attained at the center of the interface layer and average power will be around $3 \times 108 \mathrm{~W} / \mathrm{m} 3$. During experimentation, $900 \mathrm{~W}$ power microwaves has been used for bulk copper joining, it was reported that nearly $60-70 \%$ of the input power will be absorbed by the material. The simulated result on power distribution is in good agreement with experimental results [Srinath et al, 2011]. It is also observed that the maximum power will be distributed at the centre of the interface layer and it is a clear indication of volumetric heating during microwave processing. Selective heating has been observed at the sandwich layer. This selective heating can be attributed to - conductivity $(\sigma)$, permittivity $(\varepsilon)$ and permeability $(\mu)$ loss mechanisms of interface layer. The sandwich layer had developed a maximum loss of $6.069 * 10^{8} \mathrm{~W} / \mathrm{m}^{3}$. This power loss was coupled to heat transfer domain to obtain the temperature distribution. In order to find the imaginary part of permittivity of sandwich layer, an iterative process was followed. A microwave power of $900 \mathrm{~W}$ was used for bulk metal joining during the trials. It was reported that nearly $60-70 \%$ of the input power would be absorbed by the material [National Research Council, 1994]. The simulated result on power distribution at the concentrated area is a good example for the selective heating, which is one of the significance of microwave processing. The voltage reflection coefficient at the waveguide port, S11, was evaluated to be about $-4.98778 \mathrm{~dB}$, indicating that the interface layer absorbs microwave power and heats up to melting point. Due to the high thermal conductivity of the copper, the heat distribution is very fast and the temperature profile after 300 seconds has a strong peak in the center as shown in Fig.4. While heating the interface layer further, the temperature at the centre of the interface layer eventually reaches to its melting point and subsequently wetting of the bulk copper interfaces, thus joining will take 
place. The nonlinear effects of microwave absorption and heat conduction model during heating of the interface powder and bulk interfaces have been observed. The temperature profile in the Fig. 4 shows the maximum temperature is attained at the centre of the interface layer and bulk interface surfaces. Further, it is observed that maximum temperature of $1019^{\circ} \mathrm{C}$, which is a melting temperature of copper, has been attained after $300 \mathrm{~s}$.

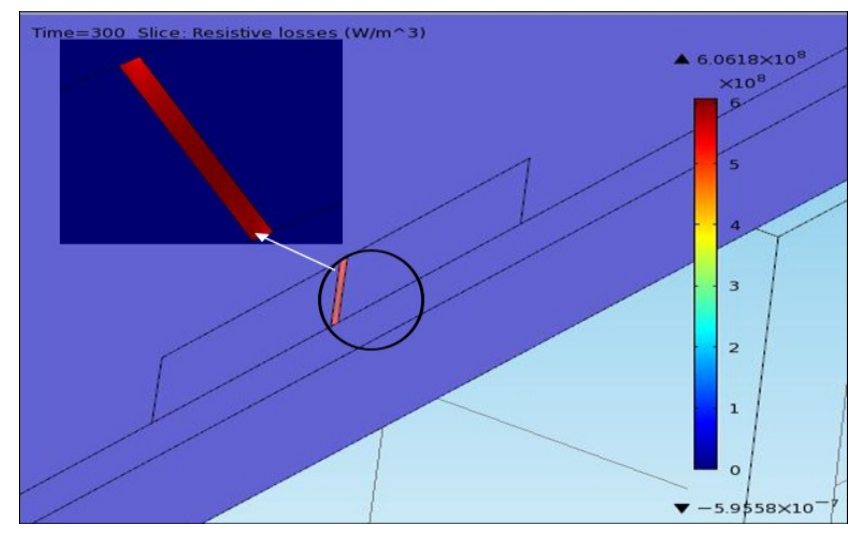

Fig. 3 Dissipated microwave power distribution; Inset: zoomed view of interface area

The maximum power observed at the joint interface is $6.0618 \times 10^{8} \mathrm{~W} / \mathrm{m}^{3}$, however, the temperature will be around $1144^{\circ} \mathrm{C}$ was attained in $300 \mathrm{~s}$ in copper joints. It is worth mentioning here that, during experimentation, the copper joints of similar configurations were obtained in $300 \mathrm{~s}$. There is nearly $8 \%$ variation in simulated results with the experimental results in copper joints. This indicates that the simulation results are in good agreement with the experimental findings [Sharma et al, 2009; Srinath et al, 2011]. The time-temperature profiles show the characteristics of microwave heating. The effect of temperature during microwave heating of materials along the joint as well as across the joints was also studied.

Figure 5 illustrates time temperature curve during MW heating of copper joint. . It is observed from the Fig. 5 that, upto about 25 $\mathrm{s}$, the increase in temperature is non-linear, indicating poor microwave interaction with metals in hybrid mode of heating. Once the metal reaches the observed critical temperature $\left(\sim 180^{\circ} \mathrm{C}\right)$, the rise in temperature is almost linear as seen in Fig. 5. This indicates that at room temperature the interaction of microwaves with metallic materials is very poor. As the metallic material reaches an elevated temperature, microwaves start coupling with the metallic material which is clearly observed from the results presented in Fig. 5. For a loss factor of 0.055 of real permittivity 3.391, the preset value of temperature was obtained as $1019{ }^{0} \mathrm{C}$. Temperature distribution with respect to time during the exposure period was obtained. Temperature along the length and depth of the specimen indicated that the maximum temperature was concentrated at the core of the interface layer. Reversible temperature gradient, which is a characteristic feature of microwave heating, was observed owing to microwave heating. The electrical field distribution showed coupling is maximum at the interface layer. The simulated results showed that the estimated temperature is attained in $300 \mathrm{~s}$. Thus, it is possible to estimate microwave processing behavior of metallic materials.

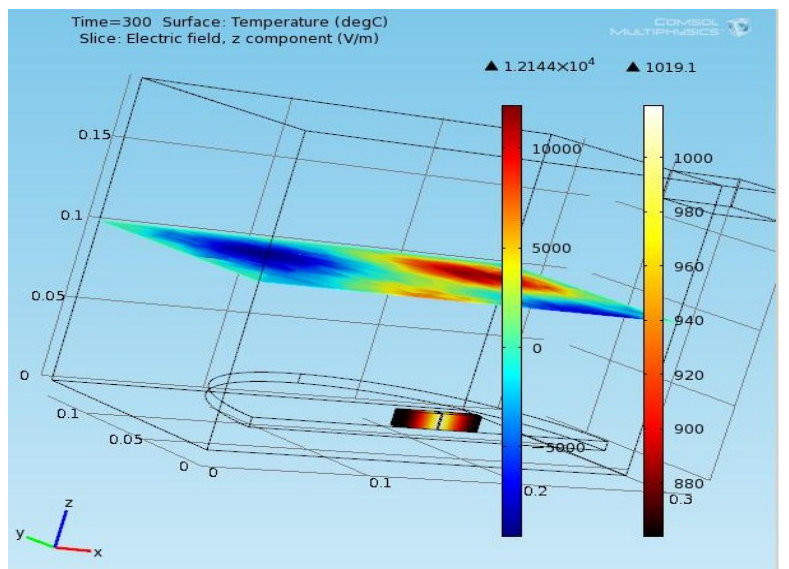

Fig. 4 Temperature distribution at the interface layer in the bulk copper joint during microwave heating 


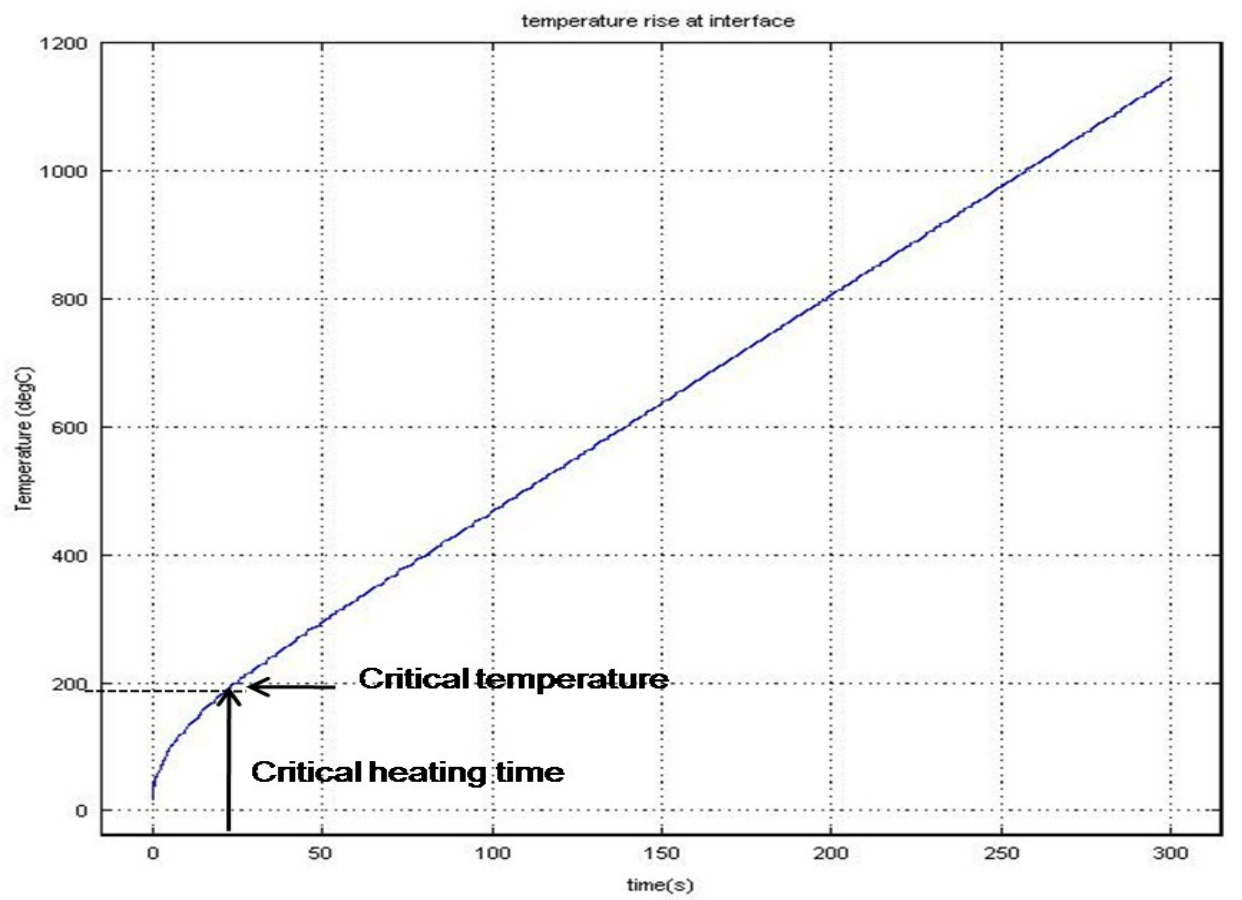

Fig. 5 Typical time temperature characteristics during MW heating of copper joint

\section{Conclusions}

It is clear from the above results that the $\mathrm{E}$ field is more important than the $\mathrm{H}$ field during microwave heating of metals. The simulation results are in good qualitative and quantitative agreement with the experimental findings. During microwave heating both $\mathrm{E}\left(\mathrm{TE}_{10}\right)$ and $\mathrm{H}\left(\mathrm{TM}_{01}\right)$ fields contribute to the heating; for different materials, however, there may be only one of them which dominate the heating process during microwave exposure. There is no control on selection of modes of microwaves in the software tool. One has to select either $\mathrm{TE}_{10}$ or $\mathrm{TM}_{01}$ mode only. Several attempts were made during simulation, however, the reported results obtained for $\mathrm{TE}_{10}$ mode only. It should be pointed out that the microwave heating of materials is very sensitive to the values of permittivity ( $\varepsilon r)$ and permeability $\left(\mu_{r}\right)$ parameters. Accordingly, the influence of these two parameters could also be clearly observed in the simulation results through the COMSOL tool. In the present study importance of $E$ field and its significance effect has been studied for microwave interaction of copper material. Due to the unavailability of permeability values, only the effect of permittivity $\left(\varepsilon_{\mathrm{r}}\right)$ is taken in this model. In the heat transfer module only the effect of conduction is taken into account. For the future study, the other modes of heat transfer can be included. The work can be extended to different material configuration,

\section{Nomenclature}

E-field: Electric field

H-field: Magnetic field

TE: $\quad$ Transverse Electric

TM: Transverse Magnetic

CuO: $\quad$ Copper oxide

$\mathrm{P}_{\text {in }}$ : Input Power

\section{References}

Agrawal D, Roy R, Cheng J, and Gedevanishvili S, 1999, Full sintering of powdered metal parts in microwaves, Nature, Vol. 399, pp. 668-670.

Ayappa KG, Davis HT, Crapiste G, Davis EA, Gordon J, 1991, Microwave heating: an evaluation of power formulations, Chemical Engineering Science, Vol. 46, pp. 1005-1016,

Chaterjee K, Das D and Chakravorthy D, 2005, Optical absorption in composites containing copper core- copper-oxide shell nanostructure in a silica gel, J.Phys: D Appl Phys, Vol. 38, pp. 451-455,.

Chatterjee A, Basak T, Ayappa KG, 1998, Analysis of microwave sintering of ceramics, AIChE Journal, Vol. 44, pp. $2302-2311$.

Clark DE, Sutton WH and Lewis D, 1996, Microwave processing of materials, Annu Rev Mater Sci., Vol. 26, pp. $299-01$. 
Ma J, Smit, CT, Weisel GJ, Weiss BL, Miskovsky NM, Zimmerman DT, 2006, Single mode microwave heating of copper powder metal compacts, Proceedings of the COMSOL Users Conference Boston.

Olevsky EA, 1998, Theory of sintering: from discrete to continuum, Materials Science and Engineering, Vol. 23, pp. 41-100.

Sharma AK, Aravindan S and Krishnamurthy R, 2001, Microwave glazing of alumina-titania ceramic composite coatings, Matt Let; Vol. 50, pp. 295-01.

Sharma AK, Srinath MS and Pradeep Kumar, 2009, Microwave Joining of Metallic Materials, Indian Patent, (Application No. 1994/Del/2009).

Srinath M.S., Sharma A.K. and Kumar P., 2011, A new approach to joining of bulk copper using microwave energy, Materials and Design, Vol. 32, No. 4, pp. 2685-2694.

Sutton W.H., 1989, Microwave processing of ceramic materials, The American Ceramic Society Bulletin, Vol. 168, pp. 376-86.

Duan Y., Dan C, Sorescu and Karl J.J, 2006, Finite element approach to microwave sintering of oxide materials, Proceedings of the COMSOL Users Conference Boston.

\section{Biographical notes}

Dr. M S. Srinath is currently working as Associate Professor in the Department of Mechanical Engineering, Malnad College of Engineering, Hassan, Karnataka, India. He obtained his B.E. in Industrial \& Production Engineering from Mysore University, India and Master's Degree from National Institute of Technology Karnataka, Surathkal, India and later on Ph.D. from IIT Roorkee, Roorkee, India. He has twelve years of teaching, three years of research and two years of industrial experience. He has published four papers in international journals and fifteen papers in various international and national conferences. He has filed an Indian patent based on his research outcome. His areas of interest are microwave material processing, advance manufacturing processes and industrial engineering. $\mathrm{He}$ is member of IE and ISTE.

Mr. P Suryanarayana Muthty currently working as an Engineer in SAIL Ranchi. He has done his B.E., in Mechanical Engineering from JNTU Hyderabad and later on he did his Masters program in Production Engineering from IIT Roorkee.

Dr. Apurbba Kumar Sharma, currently Assistant Professor in the Department of Mechanical \& Industrial Engineering at the Indian Institute of Technology Roorkee, Roorkee. He obtained his B.E. in Mechanical Engineering from Jorhat Engineering College, Assam and his Master's and Doctoral Degrees from Indian Institute of Technology Madras, Chennai. He has 18 years of teaching experience and currently supervising five Ph.D. theses. He has published more than 60 research papers in reputed international and national journals and various conferences. He has one Indian Patent to his credit and filed four more Indian patents recently. Currently, he has been the Principal Investigator for four externally funded projects for around seventy lakh rupees. He is a reviewer to various international journals. His research areas include - advanced manufacturing processes, microwave processing of materials and surface modifications.

Prof. Pradeep Kumar is presently working as Dean, Finance \& Planning and Professor in the Department of Mechanical \& Industrial Engineering, IIT Roorkee, Roorkee. He obtained his B.E., M. E. and Ph.D. from the then University of Roorkee (Now IIT Roorkee). He has twenty years of Teaching and Research experience and guided twenty one Ph.D thesis; eight Ph.D. thesis are in progress. He has worked as visiting Assistant Professor in West Virginia University, U.S.A.; Research Associate, Wayne State University, U.S.A. and as Visiting Professor at AIT Bangkok. He has published more than 350 research papers in reputed international and national journals and various conferences and filed two Indian patents. He has completed 33 consultancy project and 14 sponsored research projects of various organizations in India and abroad. He is reviewer of various international journals and editorial board member of international journals. His research interests include Supply Chain Management (SCM), Advanced Manufacturing Processes; Microwave Joining of Metals, Metal Casting; Industrial Engineering; Quality Engineering: Robust Design Methodologies, Reliability Engineering; and Production \& Operations Management.

Dr. M V Kartikeyan is presently working as Professor in the Department of Electronics \& Computer Engineering, IIT Roorkee, Roorkee. He worked aas research scientist at CEERI, Pilani, India and IHM-KIT, Germany. He is a recipient of Alexander Von Homboldt Felowship. He has guided more than 7 Ph.Ds and about 14 M.Tech. He has published more than 200 research papers and authored two books in the area of Microwave Engineering. He is a member of professional bodies like IEEE, IETE, IE and VEDA.

Received January 2012

Accepted June 2012

Final acceptance in revised form August 2012 